\title{
Competencias laborales de personas con discapacidad física, egresadas del instituto de rehabilitación infantil (iri) de Santiago entre 1990-1991.primera parte
}

\author{
Navarrete Valdebenito, Mabel; Díaz Ortíz, Mauricio
}

\begin{abstract}
- Abstract
In 1995 the Vocational Orientation and Work Integration Program of the Institute de Rehabilitation de Santiago (Children Rehabilitation Institute of Santiago) revised the files of patients that were discharged from the Institute, between the years 1990 and 1991 due to reaching the limit age of acceptance of 18 years. From this a group classification was made according to three variables: independence level, qualifications and cognitive level. A job prognosis was assigned to each of these groups which was called "work capability". The ones with the best prognosis were independent in self care, had finished high school or superior education and didn't have cognitive damage. After ten years a follow up was done to find out about the current situation of these people.
\end{abstract}

The purpose of this study is to contribute in the creation of action strategies for the Vocational Orientation and Work Integration Program and to be baselines for early intervention in children and their families to achieve the final purpose of rehabilitation: full integration in society.

This study was designed in two parts according to the main goals. The first part was made as a quantitative study and the second part as a qualitative study. This article presents the results of the first part.

\section{- Resumen}

En el año 1995 el Programa de Orientación Vocacional e Integración Sociolaboral del Instituto de Rehabilitación Infantil de Santiago, realizó un estudio de fichas de pacientes dados de alta por edad en los años 1990 y 1991, resultando de ello una clasificación por grupos según tres variables, nivel de independencia, formación y nivel cognitivo. A cada uno de estos grupos se le asignó un pronóstico laboral, llamado "capacidad para el trabajo". Aquel de mejor pronóstico era el que presentaba independencia en autocuidado, con enseñanza media completa o superior y sin daño cognitivo. Transcurridos 10 años, se decide realizar un estudio de seguimiento de estos casos para conocer la situación actual de estas personas.

El propósito de este estudio es realizar un aporte para la elaboración de nuevas estrategias de acción del Programa de Orientación Vocacional e Integración Laboral y servir de base para las líneas de acción temprana en niños, jóvenes y sus familias para lograr el propósito final de la (re) habilitación: una integración plena a la sociedad.

Este estudio fue diseñado en dos partes según los objetivos generales. La primera parte se realizó mediante las características de investigación cuantitativa y la segunda está diseñada con un enfoque cualitativo. Este artículo presenta los resultados de la primera parte. 


\section{- Introducción}

Para el Instituto de Rehabilitación Infantil de Santiago (IRI), siempre ha estado entre sus propósitos prestar el mejor servicio a las personas que atiende, de acuerdo a los recursos materiales y tecnológicos que posee y al nivel de sus profesionales y técnicos. Consecuentemente, implementa nuevas formas y modalidades de intervención dirigidas a la (re) habilitación integral de niños y jóvenes con discapacidad, en la perspectiva de trabajar todos los aspectos de los pacientes para que se proyecten a una vida activa con distintos grados de participación, según las condiciones que presentan. Las personas con discapacidad al ser parte de la sociedad tienen derechos y entre estos, el derecho a la participación en la vida económica del país, la que sólo será posible en la medida de la realización de una actividad productiva.

Si consideramos actividad productiva como aquella que habitualmente se realiza bajo condiciones corrientes o especiales, siempre que represente un ingreso económico ${ }^{(1)}$, apreciamos que los jóvenes con discapacidad física, atendidos en el IRI con compromiso funcional leve o moderado, se encuentran en condiciones de incorporarse a un empleo formal, siempre y cuando la formación alcanzada les haya aportado condiciones mínimas para desempeñarse en una actividad de trabajo y no existan otras condicionantes que lo impidan. Asimismo, no podemos desconocer que un porcentaje importante de la población atendida en esta Institución, debido a su severo compromiso funcional, siempre necesitarán condiciones especiales para el desarrollo de una actividad productiva.

A través de la experiencia del programa de Orientación Vocacional e Integración Laboral, se constata las dificultades que enfrentan los jóvenes que son atendidos ${ }^{(\underline{b})}$, para desempeñar una actividad de trabajo (empleo formal, trabajo independiente, trabajo en condiciones especiales, etc.). En este sentido, conocer las características relacionadas con el aprendizaje y desempeño de una actividad productiva resulta relevante para el futuro diseño de las acciones y sus correspondientes actividades.

Con el afán de objetivar y sistematizar el trabajo en esta área, el equipo que trabajaba en rehabilitación profesional en el año 1995, realizó un estudio que contempló la revisión de 141 fichas de pacientes dados de alta por edad en los años 1990 y 1991. Uno de los productos de este estudio, lo constituyó la confección de categorías que pronosticaran las posibilidades para desempeñar un trabajo a la población que se atiende en el Instituto de Rehabilitación Infantil de Santiago. Estas categorías se denominaron "Grupos de Capacidad para el Trabajo" y se definieron según tres variables: nivel de independencia, nivel de estudios y capacidad intelectual ${ }^{(2)}$.

La combinación de las categorías resultantes según niveles de estas variables, permitió agrupar estos 141 casos en 8 grupos, cada uno de los cuales, tendría un determinado pronóstico laboral (Tabla 1).

Tabla 1: Grupos de Capacidad para el Trabajo (1995)

\begin{tabular}{|c||c|c|l|}
\hline Grupos & Capacidad intelectual & $\begin{array}{c}\text { Grado de } \\
\text { Independencia }\end{array}$ & \multicolumn{1}{|c|}{ Tipo de Educación } \\
\hline 1 & Normal & Independiente & $\begin{array}{l}\text { Educación media completa. } \\
\text { Técnico profesionalUniversitaria } \\
\text { cursando/incompleta }\end{array}$ \\
\hline \hline
\end{tabular}

De buen pronóstico laboral. Con posibilidades de capacitación técnica profesional o universitaria. Podrán optar a empleos semicalificados y calificados, de tipo técnico o profesional. Es un grupo que puede hacer uso de becas o facilidades de capacitación y/o formación existentes. 


\begin{tabular}{|c|c|c||c|}
\hline Grupos & $\begin{array}{c}\text { Capacidad } \\
\text { intelectual }\end{array}$ & Grado de Independencia & Tipo de Educación \\
\hline 2 & Normal & Independiente & $\begin{array}{c}\text { Básica completa o } \\
\text { incompleta }\end{array}$ \\
\hline \hline
\end{tabular}

De buen pronóstico laboral. Con posibilidades de capacitación en oficios pudiendo aprovechar tanto los aspectos teóricos y prácticos de la enseñanza. El empleo será semicalificado o no calificado, con posibilidades de promoción en el empleo

\begin{tabular}{|c|c|c|c|}
\hline \hline Grupos & $\begin{array}{c}\text { Capacidad } \\
\text { intelectual }\end{array}$ & $\begin{array}{c}\text { Grado de } \\
\text { Independencia }\end{array}$ & Tipo de Educación \\
\hline 3 & Limítrofe & Independiente & $\begin{array}{c}\text { Media o básica completa o } \\
\text { incompleta }\end{array}$ \\
\hline \hline
\end{tabular}

Capacitación práctica en oficios. Con posibilidades de empleo en puestos de trabajo semicalificados. Es un grupo que tiene mayores posibilidades en este tipo de puestos, comparado con aquellos de $\mathrm{Cl}$ inferior.

\begin{tabular}{|c||c||c||c|}
\hline \hline Grupos & Capacidad Intelectual & Grado de Independencia & Tipo de Educación \\
\hline 4 & Limítrofe o RM leve & Independiente & Enseñanza Diferencial \\
\hline \hline
\end{tabular}

Capacitación práctica en oficios. Empleo no calificado (RM leve) o semicalificado (limítrofe). Sin posibilidades de acceder a empleo calificado.

\begin{tabular}{|c|c|c||c|}
\hline \hline Grupos & Capacidad intelectual & Grado de Independencia & Tipo de Educación \\
\hline 5 & Normal & Dependiente & Media completa o cursando \\
\hline \hline
\end{tabular}

Pronóstico variable. Según las condiciones socioeconómicas y ambientales, que influyen en las posibilidades de superar algunas discapacidades. Capacitación técnica o formación técnica profesional y universitaria. Podrán tener empleos calificados, técnicos o profesionales siempre que se logren superar discapacidades específicas.

\begin{tabular}{|c|c|c|c|}
\hline \hline Grupos & Capacidad intelectual & Grado de Independencia & Tipo de Educación \\
\hline 6 & Normal & Dependiente & Básica completa o incompleta \\
\hline \hline
\end{tabular}

Regular, variable. Depende de cada caso. La dependencia de terceros limita las posibilidades de este grupo. Es necesario una adecuada evaluación para definir la capacitación que podría lograr. Están en condiciones de realizar trabajos en su casa o cercanos a ella o bien bajo condiciones especiales para traslado o manejo en el baño.

\begin{tabular}{|c|c|c|c|}
\hline \hline Grupos & Capacidad intelectual & Grado de Independencia & Tipo de Educación \\
\hline 7 & RM leve o moderado & Dependiente & Enseñanza diferencial \\
\hline \hline
\end{tabular}

Mal pronóstico laboral. Se puede realizar capacitación en manualidades, con posibilidades de realizar trabajos domiciliarios sencillos y supervisados. Candidato a taller protegido, no se cuenta con recursos comunitarios para este grupo.

\begin{tabular}{|c|c|c||c|}
\hline Grupos & Capacidad intelectual & $\begin{array}{c}\text { Grado de } \\
\text { Independencia }\end{array}$ & Tipo de Educación \\
\hline \hline 0 & $\begin{array}{c}\text { RM moderado severo o } \\
\text { grave }\end{array}$ & Dependiente & $\begin{array}{c}\text { Ssin escolaridad o } \\
\text { diferencial }\end{array}$ \\
\hline Sin capacidad para el trabajo & \\
\hline
\end{tabular}

De los pacientes clasificados en alguna de estas categorías, no se tiene información desde 1995. Después de 10 años, se quiere comprobar el valor pronóstico de estos "Grupos de Capacidad para el Trabajo" y describir las competencias laborales que han permitido a personas con discapacidad física el desempeño de una actividad productiva. Los objetivos específicos son: conocer la situación laboral actual de estas personas, conocer la influencia de las variables (consideradas en 
el estudio) para la integración socio-laboral, describir las competencias laborales para adquirir y mantenerse una actividad productiva y valorar la experiencia de aquellas personas con discapacidad que han logrado desempeñarse en el mercado de trabajo.

(b) Actualmente la población activa a nivel nacional es de 21.675 pacientes, con una cobertura de un $75 \%$ a nivel nacional en menores de 18 años. En Santiago, la población activa es de 8.830 casos, correspondientes a personas que han recibido atención en los últimos 5 años. También existe una población pasiva en este Instituto que se acerca a las 13.500 personas, las cuales están dadas de alta, ya sea por edad (a los 18 años), ausentismo o fallecimiento. (Base de datos de Sistema de Gestión y Rehabilitación; Teletón, Santiago, Diciembre 2003)

\section{- Empleo Jóvenes y Discapacidad}

En el libro" Jóvenes y empleo en los noventa"(ํㅡㄹ cuales nos sirven para explicar posteriormente los resultados de este estudio. Estos son:

1. Calificación Nula: Incluye a las personas analfabetas, las que no han tenido instrucción formal y las que han alcanzado hasta tres años de educación primaria. En este estrato se considera que no se cumplen los niveles mínimos compatibles con las emergentes estructuras de ocupación.

2. Calificación Baja: Personas con niveles de educación primaria con más de tres años hasta niveles básicos de secundaria. Se incluye a las personas con niveles mínimos para comenzar una experiencia laboral en los sectores modernos del empleo. Es un segmento heterogéneo, pero donde se cuenta con una base mínima de aprendizajes para comenzar una formación polivalente compatible con los nuevos requerimientos de la ocupación.

3. Calificación Media: personas con más de tres años de educación de nivel secundario, se trata de personas que han terminado el ciclo básicos de aprendizajes para proseguir estudiando o trabajando en ocupaciones con mayores requisitos en cuanto a niveles de abstracción y comprensión.

4. Calificación Técnica: Se incluyen las personas que han realizado educación técnico formal.

5. Calificación Superior: Individuos con formación terciaria, ya sea universitaria o no universitaria.

Esta clasificación de los niveles de calificación de la población, ha sido realizada teniendo en cuenta una perspectiva comparativa a nivel latinoamericano, sin embargo, se deben considerar los estándares de calidad educativa en los diferentes países.

Los países de América Latina presentan variaciones considerables en cuanto a riqueza, grado de desarrollo y funcionamiento del mercado laboral. El crecimiento del empleo en el sector informal es motivo de especial inquietud en esta región. Se calcula que el $85 \%$ de todos los nuevos puestos de trabajo se crean en la economía informal, sólo en Chile y Colombia descendió el empleo en ese sector durante el decenio de 1990. ${ }^{(4)}$

La experiencia acumulada en el Instituto de Rehabilitación Teletón de Santiago, nos muestra la importancia que adquieren los estudios de enseñanza media para acceder a un puesto de trabajo. Durante el año 2002 las ofertas de empleo, estuvieron dirigidas mayoritariamente a personas con un grado de formación de a lo menos enseñanza media completa. ${ }^{(5)}$

De acuerdo a lo señalado, los siguientes son los puestos de trabajo a los que accedieron mayoritariamente los jóvenes durante 2002: Operador telefónico; operador de atención a clientes; 
vendedor telefónico; empleado administrativo; secretaria, digitador, cajero, monitor de cursos a distancia.

A diferencia de lo observado en la primera mitad de la década de los años 90 , en que se recibieron mayoritariamente ofertas para puestos de trabajo que no precisaban de estudios completos de enseñanza media, desde 1996 es un requisito indispensable el haber cursado estudios de enseñanza media.

Se aprecia que las oportunidades de empleo a las que se accede como Institución, podrían están relacionadas con la tasa de desempleo en la Región Metropolitana en el periodo correspondiente. Es así que en periodos de desempleo menor, se accede a un mayor número de ofertas de empleo

\section{- Metodología}

Este estudio corresponde a uno de tipo descriptivo, retrospectivo.

El universo es de 141 casos de jóvenes con discapacidad física que participaron en el estudio de 1995 y que fueron dados de alta por edad en los años 1990 y 1991.

La muestra está representada por los casos que pudieron ser contactados en el año 2003, no se consideraron criterios de exclusión. Se entrevistaron 29 casos, lo que corresponde al $20 \%$ del total de fichas revisadas en el año 1993.

Considerando el bajo número de sujetos que resultó posible encuestar, se fusionaron los ocho grupos de Capacidad para el Trabajo y reagruparon en cuatro categorías (Tabla 2). La nueva reagrupación se realizó considerando dos variables: nivel cognitivo y grado de independencia, estas eran similares en los nuevos grupos lo que no modificó profundamente los criterios utilizados con anterioridad. Respecto de la variable estudios no se diferenció por nivel cursado, ya que todos los grupos presentaban nivel de escolaridad, exceptuando el grupo D.

Tabla 2. Nueva Clasificación de Grupos de Capacidad para el Trabajo

\begin{tabular}{|l|l|l|l||l|}
\hline \multicolumn{1}{|c|}{ Variables } & \multicolumn{1}{c|}{ Grupo A } & \multicolumn{1}{c|}{ Grupo B } & Grupo C & \multicolumn{1}{|c|}{ Grupo D } \\
\hline $\mathrm{Cl}$ & Normal & $\begin{array}{l}\text { Limítrofe o DM } \\
\text { leve }\end{array}$ & Normal & $\begin{array}{l}\text { DM Leve- } \\
\text { Moderada- } \\
\text { Severa }\end{array}$ \\
\hline $\begin{array}{l}\text { Grado de } \\
\text { Independencia }\end{array}$ & Independientes & Independientes & Dependientes & Dependientes \\
\hline Estudios & $\begin{array}{l}\text { Básicos, Medios, } \\
\text { Superiores }\end{array}$ & $\begin{array}{l}\text { Diferencial- } \\
\text { Básicos-Medios }\end{array}$ & $\begin{array}{l}\text { Básicos- } \\
\text { Medios }\end{array}$ & $\begin{array}{l}\text { Sin estudios- } \\
\text { Diferencial }\end{array}$ \\
\hline
\end{tabular}

La recolección de datos se realizó principalmente mediante entrevista telefónica, en 4 casos mediante entrevista personal y en uno de ellos se recibió respuesta vía correo electrónico. En cuatro casos la información fue entregada por un familiar directo, debido al grado de compromiso del sujeto.

El instrumento de recolección de información permitió registrar datos generales de cada sujeto, nivel de estudios, nivel capacitación o formación, historia laboral y apreciación respecto de factores considerados como facilitadores o dificultadores para el logro de un empleo. 
Se incorporó una pregunta de ingreso económico familiar, siendo difícil obtener la información, por lo cual no pudo ser analizada posteriormente.

A continuación se presentan los resultados del análisis descriptivo de la información recopilada.

\section{- Resultados}

Del $20 \%$ encuestado se logra obtener la siguiente información: Se encuestan 18 casos que corresponden al grupo A, 10 al B, 1 al D y no se realizaron encuestas en el Grupo C.

La muestra por sexo se distribuyó de la siguiente manera: $49 \%$ mujeres y $51 \%$ hombres.

El rango de edad de los encuestados se encuentra entre los 28 a 33 años, siendo mayor en número en el rango 28-30 años.

Según la clasificación utilizada en la encuesta CASEN del año 2000 (6), actualmente el $55.5 \%$ del grupo A está empleado, $27,7 \%$ desempleado y un 16,8\% inactivo. Respecto del grupo B un 10\% trabajaba, un $20 \%$ estaba desempleado y un $70 \%$ inactivo (Ver Gráfico 1).

\section{Gráfico 1. Situación Laboral}

\section{Situación Laboral}

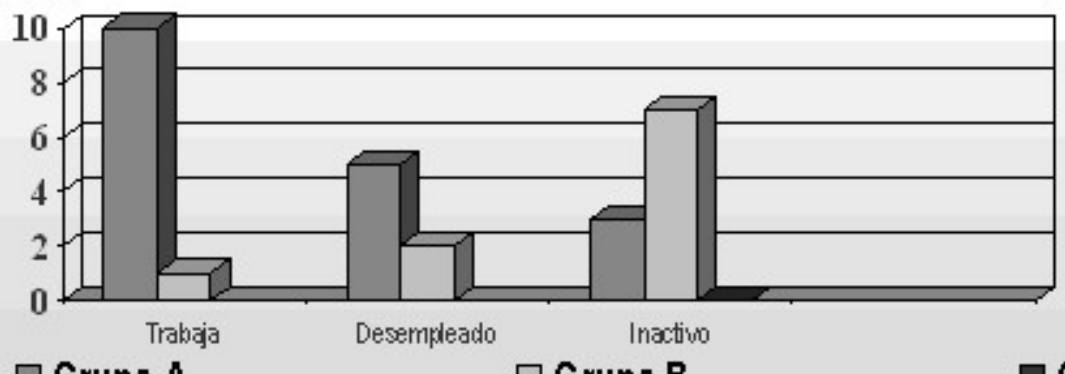

Grupo A

Grupo B

Grupo D

De los 11 casos que trabajan, de ambos grupos, cinco lo hacen directamente en una actividad de trabajo relacionada con la formación profesional recibida. En los otros cinco casos, la actividad 
laboral desempeñada se relaciona con el nivel de estudios y sólo en un caso la persona realiza una actividad laboral para la cual tiene un nivel de formación técnico de mayor calificación.

En relación al nivel de estudios, los grupos se distribuyen como se muestra en el gráfico 2. En el grupo A, 10 de las personas lograron formación superior, siete alcanzaron enseñanza media científica humanista o técnico profesional y una persona sólo enseñanza básica completa. En el grupo B en nivel de formación se concentra en educación diferencial (60\%) enseñanza básica $(30 \%)$ y un $10 \%$ en enseñanza media. La persona del grupo D no logró integración escolar.

\section{Gráfico 2. Nivel de Estudios}

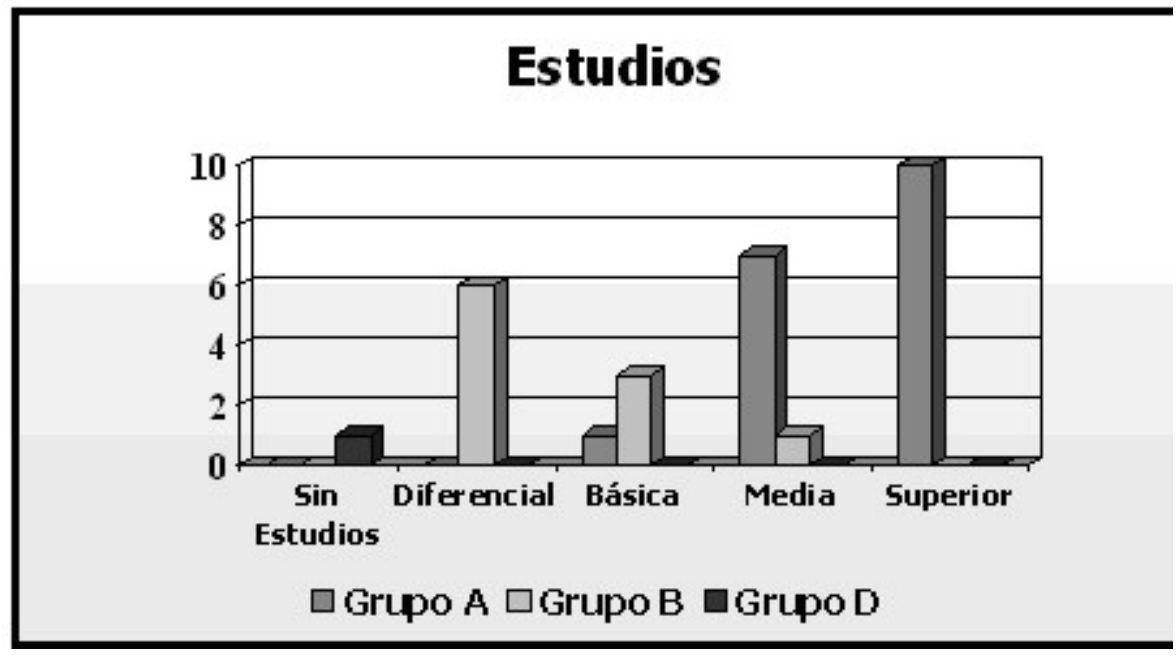

En la tabla 3 se aprecia la valoración que hacen de factores personales para la obtención y permanencia en un trabajo las personas de los grupos A y B. Destaca en el grupo A la preponderancia dada a aquellos factores que dependen de la propia persona (perseverancia, hábitos de trabajo, autonomía y establecerse metas).

Tabla 3

\begin{tabular}{|l|c||c|}
\hline \multicolumn{1}{|c||}{ Variables } & Grupo A & Grupo B \\
\hline Nivel de Formación/capacitación & $67 \%$ & $40 \%$ \\
\hline Perseverancia & $83 \%$ & $50 \%$ \\
\hline \hline Hábitos de trabajo & $83 \%$ & $50 \%$ \\
\hline Autonomía/Independencia & $67 \%$ & $50 \%$ \\
\hline \hline Establecimiento de metas & $78 \%$ & $40 \%$ \\
\hline
\end{tabular}

Respecto a considerar ciertas barreras o condiciones para la integración y permanencia en un empleo, son las urbanísticas, del transporte, nivel de integración alcanzado y los aspectos integración, las que las personas consideran con mayor frecuencia como barrera. En la Tabla 4 se explica en porcentajes la importancia que le dan estas personas a las variables mencionadas.

Tabla 4

\begin{tabular}{|l|l|l|}
\hline Variables & Grupo A & Grupo B \\
\hline
\end{tabular}




\begin{tabular}{|l|c|c|}
\hline Barreras del Transporte, urbanísticas,arquitectónicas & $28 \%$ & $40 \%$ \\
\hline Nivel de Formación & $39 \%$ & $20 \%$ \\
\hline Nivel de independencia, autonomía & $22 \%$ & $30 \%$ \\
\hline Aspectos socioculturales & $44 \%$ & $40 \%$ \\
\hline
\end{tabular}

\section{- Discusión}

Los resultados de este estudio se aproximan al pronóstico laboral definido para los "Grupos de Capacidad para el Trabajo" que se realizó en el año 1995. Es decir, hay variables que podrían determinar el futuro laboral, sin embargo existen personas que presentando y siendo favorecidas por estas variables, se alejan del pronóstico laboral, en los términos explicitados en este estudio.

- Resulta interesante considerar que en el grupo A el $100 \%$ de las personas ha tenido experiencia laboral, lo que comprobaría el valor pronóstico del estudio anterior. Sin embargo $28 \%$ de los encuestados se encontraba desempleado y $16 \%$ inactivo, mientras el $55 \%$ de los ellos estaba trabajando al momento del seguimiento. Por los resultados obtenidos en este grupo se puede concluir que a pesar de la presencia de variables funcionales similares, las personas han tenido experiencias de trabajo diferentes, especialmente en lo relacionado con la permanencia en un empleo.

- Es necesario prestar atención al valor que le atribuyen a los factores de tipo personal los individuos del grupo A, para obtener y permanecer en un empleo, a la hora de definir contenidos y competencias a trabajar en programas de entrenamiento para el trabajo en nuestra Institución.

- Considerando que en el grupo B el porcentaje de desempleo e inactividad es alto, aproximadamente el $90 \%$, se hace necesario mejorar la condición que presentan quienes pertenecen a este grupo. Al mencionar en la misma proporción los factores personales y del ambiente y su relación de oportunidades laborales, los programas de entrenamiento y asesoría para el trabajo deben contemplar actividades e intervenciones que consideren estos aspectos.

- Al ser para el grupo B, las barreras del transporte, arquitectónicas y socioculturales, las que mayoritariamente se perciben como obstáculos para su integración laboral (podría relacionarse con la presencia de un mayor compromiso motor y cognitivo), es necesario trabajar con mayor intensidad este aspecto, tanto con pacientes como con familiares y en la medida de lo posible proponer o diseñar medidas que compensen dificultades que presentan.

- De lo señalado, resulta importante destacar que el nivel de capacitación laboral o formación profesional logrado por las personas del grupo A, ha sido una herramienta efectiva para obtener y mantener una actividad laboral, ya que el trabajo efectuado se relaciona directamente con esta capacitación o formación (en 10 de 11 casos).

- Otro elemento importante se refiere a la continuidad y estabilidad laboral apreciada en estos ex pacientes. En la mayoría de los casos inician su experiencia de trabajo una vez terminan estudios o formación y presentan estabilidad en su historial de trabajo.

- En estudios posteriores, se podría considerar la edad de inicio de la vida laboral, dato que nos daría una información respecto a la madurez con la cual se perfilan en el mundo laboral.

- Los resultados de estado civil nos podrían mostrar el grado de inclusión/integración de las personas a la vida social y sus proyecciones familiares, ya que las condiciones funcionales sería un factor condicionante al momento de iniciar una vida en pareja. En este estudio, el porcentaje de solteros son aquellos que presentan mayor compromiso cognitivo por lo que efectivamente continúan dependiendo de terceras personas. 
- Al no ser considerados los servicios de empleo (Oficinas Municipales de Información Laboral) como medio de búsqueda, se hace necesario a quienes actúan como orientadores laborales informar adecuadamente de su existencia y mantener contactos mas permanentes con estas Oficinas a fin de asegurar un mejor servicio a las personas con discapacidad que recurren a ellas.

- Considerando nuestra realidad sociocultural, no es extraño que las redes sociales y los contactos de tipo personal resulten un medio importante para la búsqueda de trabajo. Este factor también debe ser considerado para el trabajo de información y asesoría ocupacional, ya que si bien pude ser muy útil para encontrar trabajo, en ocasiones conlleva malas prácticas y distorsiones que resultan perjudiciales.

- En el caso de los ex pacientes que corresponden al grupo D, las condiciones de integración deben ser especiales por su nivel de dependencia. Si bien, en distintos niveles se han planteado sugerencias y demandas, esto no se ha logrado especialmente a nivel público. No se cuenta con alternativas de trabajo en condiciones especiales para personas con discapacidades más complejas que presentan un potencial de desempeño productivo acorde con la realización de una actividad de trabajo.

- En el grupo C no se pueden establecer conclusiones, ya que no se logró encuestar a ningún representante de este grupo.

- ¿Qué hace la diferencia entre quienes se han integrado laboralmente y quienes no? o ¿por qué algunas personas teniendo las mismas oportunidades de integración son exitosas y otras no?. Un aporte de este estudio, da indicios respecto a la importancia de los factores de tipo personal para el éxito en la integración laboral. Por esto se deja planteada la segunda parte de este estudio con los objetivos de poder discriminar y determinar las competencias y habilidades que presentan las personas con discapacidad en una integración laboral exitosa.

\section{- Referencias}

(1) GLARP. Elementos de Autogestión y Mantenimiento en el Desarrollo de una Actividad Productiva. Documento de Trabajo. 1996

(2) ARREDONDO C. G et al. Programa de Evaluación y Orientación Vocacional. Informe y Propuesta. Instituto de Rehabilitación Infantil. Junio de 1993.

(3) DIEZ DE MOLINA R. Jóvenes y Empleo en los Noventa. OIT Cinterfor. 2001

(4) BLANCHFLOWER D. Emplear a los jóvenes: promover un crecimiento intensivo en empleo. Primera edición, OIT. 2000.

(5) DIAZ M. Informe: Experiencia de Integración Laboral en el Instituto Teletón de Santiago. Junio de 2003

(6) MIDEPLAN. Informe CASEN 2000 